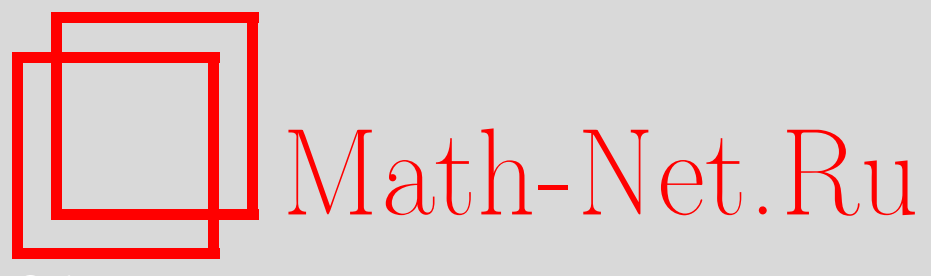

В. К. Белошапка, Представление группы голоморфных симметрий вещественного ростка в группе симметрий его модельной поверхности, Матем. заметки, 2007, том 82, выпуск 4, 515-518

DOI: https://doi.org/10.4213/mzm4019

Использование Общероссийского математического портала Math-Net.Ru подразумевает, что вы прочитали и согласны с пользовательским соглашением http://www.mathnet.ru/rus/agreement

Параметры загрузки:

IP : 54.224 .60 .19

26 апреля 2023 г., 14:20:55

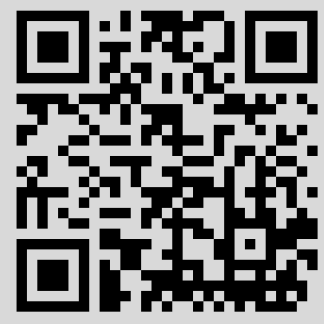


Том 82 выпуск 4 октябрь 2007

УДК 517.53

\section{Представление группы голоморфных симметрий вещественного ростка в группе симметрий его модельной поверхности}

\section{В. К. Белошапка}

Построению и изучению локальных полиномиальных моделей вещественных подмногообразий комплексного пространства посвящена серия работ. Среди основных свойств модельных поверхностей имеется следующее. Размерность локальной группы голоморфных симметрий ростка не превосходит размерности такой же группы ее касательной модельной поверхности. В данной заметке этому утверждению придается гораздо более сильная форма: связная компонента единицы группы симметрий невырожденного ростка изоморфна, как группа Ли, подгруппе группы симметрий ее касательной модельной поверхности.

Библиография: 5 названий.

Среди методов изучения вещественных подмногообразий комплексного пространства имеется метод, имеющий солидную историю, который мы называем методом модельной поверхности. Суть его в следующем. С ростком многообразия голоморфно инвариантно связывается некий образцовый, "модельный" росток того же CR-типа, нечто похожее на соприкасающийся параболоид классической дифференциальной геометрии. Эта процедура позволяет сводить вопросы о сложном объекте - произвольном ростке - к аналогичным вопросам о простом объекте - его модельной поверхности. Так или иначе изучение свойств многообразия требует выделения такого канонического объекта. (Построению и изучению локальных полиномиальных моделей вещественных подмногообразий комплексного пространства посвящена серия работ, см. [1].) Это полностью относится к вопросам о голоморфных симметриях ростка. Одним из главных характеристических свойств модельной поверхности является то, что группа ее голоморфных симметрий мажорирует в смысле размерности группу симметрий ростка. В данной заметке этому утверждению придается более правильная и сильная форма. А именно, доказывается, что связная компонента единицы в группе симметрий ростка изоморфна, как группа Ли, подгруппе симметрий ее касательной модельной поверхности.

Работа выполнена при поддержке Российского фонда фундаментальных исследований (грант № 005-01-0981) и программы "Ведущие научные школы" (грант № НШ-2040.2003.1).

(C) В. К. БЕЛОшАПКА, 2007 
В [2] было дано определение вполне невырожденного ростка типа $(n, K)$ и было показано, что полиномиальной заменой такой росток можно привести к виду

$$
\begin{aligned}
v_{2} & =\Phi_{2}(z, \bar{z})+O(3), \\
\ldots & \ldots \ldots \ldots \ldots \ldots \ldots \\
v_{\ell-1} & =\Phi_{\ell-1}\left(z, \bar{z}, u_{2}, \ldots, u_{\ell-2}\right)+O(\ell), \\
v_{\ell} & =\Phi_{\ell}\left(z, \bar{z}, u_{2}, \ldots, u_{\ell-1}\right)+O(\ell+1) .
\end{aligned}
$$

Эта запись означает, что координаты объемлющего комплексного пространства $\mathbb{C}^{n+K}$ разбиты на серии

$$
\begin{aligned}
z & =\left(z_{1}, \ldots, z_{n}\right), \\
w_{2} & =\left(w_{21}, \ldots, w_{2 k_{2}}\right), \\
\ldots & \ldots \ldots \ldots \ldots \ldots \\
w_{\ell-1} & =\left(w_{(\ell-1) 1}, \ldots, w_{(\ell-1) k_{\ell-1}}\right), \\
w_{\ell} & =\left(w_{\ell 1}, \ldots w_{\ell k}\right) .
\end{aligned}
$$

При этом локальные координаты, в которых уравнение ростка имеет такой вид, называются стандартными. Координатам пространства присвоены следующие веса: $[z]=1,\left[w_{j}\right]=j$, и тогда координаты $\Phi_{m}$ - это многочлены веса $m$, а через $O(m)$ обозначена сумма мономов веса $m$ и выше. Вещественно алгебраическая поверхность $Q=Q\left(M_{\xi}\right)$ типа $(n, K)$ в $\mathbb{C}^{n+K}=\mathbb{C}^{n} \oplus \mathbb{C}^{k_{2}} \oplus \cdots \oplus \mathbb{C}^{k_{\ell-1}} \oplus \mathbb{C}^{k}$, заданная соотношениями

$$
\begin{aligned}
v_{2} & =\Phi_{2}(z, \bar{z}), \\
& \ldots \ldots \ldots \\
v_{j} & =\Phi_{j}\left(z, \bar{z}, u_{2}, \ldots, u_{j-1}\right), \\
\ldots & \left.\ldots \ldots \ldots \ldots \ldots \ldots, u_{\ell-2}\right), \\
v_{\ell-1} & =\Phi_{\ell-1}\left(z, \bar{z}, u_{2}, \ldots, u_{\ell-1}\right) \\
v_{\ell} & =\Phi_{\ell}\left(z, \bar{z}, u_{2}, \ldots, u, \ldots\right.
\end{aligned}
$$

или просто $v=\Phi(z, \bar{z}, u)$, называется касателъной моделъной поверхностъю ростка $M_{\xi}$. Здесь координаты $\Phi_{j}$ при $j=2, \ldots, \ell-1$ - это вещественный базис (произвольный) пространства $\mathscr{C} \mathscr{H}_{j}$, а координаты $\Phi_{\ell}$ - линейно независимые элементы $\mathscr{C} \mathscr{H}_{\ell}$.

Напомним вкратце общую схему этого построения. Пусть $M_{\xi}$ - это наш росток, $n$ - комплексная размерность комплексной касательной, $K$ - вещественная коразмерность, $\ell$ - длина алгебры Леви-Танаки, тогда $n+K$ - это размерность объемлющего комплексного пространства. Тип $(n, K)$ для вполне невырожденного многообразия однозначно определяет длину. Модельная поверхность произвольного типа $(n, K)$ строится в результате рекуррентного процесса. На первом шаге переменной $z \in \mathbb{C}^{n}$ присваивается вес 1. Далее идет процесс вычисления некоторой последовательности данных. Вот список данных с номером $m$ : прямое разложение пространства многочленов веса $m$ в сумму пространства гармонических форм $\mathscr{H}_{m}$ и 
его дополнения - пространства нормализованных многочленов веса $m-\mathscr{C} \mathscr{H}_{m}, k_{m}-$ размерность пространства $\mathscr{C} \mathscr{H}_{m}$, пространство $\mathbb{C}^{k_{m}}$ с координатами $w_{m}=u_{m}+i v_{m}$ веса $m$. Процесс заканчивается на $\ell$-м шаге, где $\ell$ - длина алгебры Леви-Танаки. Условием окончания процесса является попадание коразмерности $K$ в диапазон

$$
k_{2}+\cdots+k_{\ell-1}<K \leqslant k_{2}+\cdots+k_{\ell}
$$

При этом избыток коразмерности $k=K-\left(k_{2}+\cdots+k_{\ell-1}\right)$ может изменяться в пределах от 1 до $k_{\ell}$.

Пусть aut $M_{\xi}$ - это алгебра Ли ростков векторных полей в точке $\xi$ касательных на $M_{\xi}$ к $M_{\xi}$ с голоморфными коэффициентами, Aut $M_{\xi}$ совокупность ростков голоморфных отображений в точке $\xi$, порожденных полями из aut $M_{\xi}$. Эти отображения, очевидно, переводят росток $M_{\xi}$ в себя. Пара aut $M_{\xi}$ и Aut $M_{\xi}-$ это поля, обращающиеся в нуль в точке $\xi$ и порожденные ими отображения, сохраняющие $\xi$ неподвижной.

Так как вполне невырожденный росток является ростком конечного типа, то в соответствии с [3] можно утрверждать, что Aut $M_{\xi}$ является группой Ли в естественной топологии. Причем многообразие параметров, задающих отображения из $\operatorname{Aut}_{\xi} M_{\xi},-$ это поверхность в пространстве струй определенного порядка.

Если росток записан в стандартных координатах, то $\xi$ - это начало координат в $\mathbb{C}^{n+K}$, и мы пишем aut $M_{0}$ и Aut $_{0} M_{0}$. Соответственно мы можем рассмотреть группу и алгебру модельной поверхности $-\operatorname{aut}_{0} Q_{0}$ и $\mathrm{Aut}_{0} Q_{0}$.

Элементы aut $M_{0}$ и aut $Q_{0}$ - это векторные поля, которые в стандартных координатах могут быть записаны в виде

$$
X=2 \operatorname{Re}\left(f_{1} \frac{\partial}{\partial z}+f_{2} \frac{\partial}{\partial w_{2}}+\cdots+f_{\ell} \frac{\partial}{\partial w_{\ell}}\right),
$$

где $f_{1}, \ldots, f_{\ell}$ голоморфны и обращаются в нуль в начале координат. Полагая $\left[\partial / \partial w_{j}\right]=-j$, мы распространим градуировку на векторные поля. Получаем разложение в сумму весовых компонент $X=\sum X_{j}$, при этом aut $M_{0}$ становится градуированной алгеброй Ли вида aut $_{0} M_{0}=\sum g_{j}$.

В [2] были доказаны следующие утверждения:

(a) если aut $M_{0}=\sum g_{j}$, то $g_{j}=0$ при $j<0$;

(b) существует число $D$, зависящее, вообще говоря, от типа $(n, K)$, такое, что если $X \in \operatorname{aut}_{0} Q_{0}$, то при $j>D X_{j}=0$;

(c) если $X=X_{0}+\cdots+X_{D} \in$ aut $_{0} Q_{0}$, то каждая весовая компонента $X_{j} \in$ aut $_{0} Q_{0}$;

(d) если $X=X_{0}+\cdots+X_{D}+\cdots \in$ aut $_{0} M_{0}$, то $\widehat{X}=X_{0}+\cdots+X_{D} \in$ aut $_{0} Q_{0}$;

(е) если $X=X_{0}+\cdots+X_{D}+\cdots$ и $Y=Y_{0}+\cdots+Y_{D}+\cdots \in$ aut $_{0} M_{0}$, причем $X_{0}=Y_{0}, \ldots, X_{D}=Y_{D}$, то $X=Y$.

Определим отображение $\psi$ из aut $_{0} M_{0}$ в aut $_{0} Q_{0}$ следующим образом:

$$
\psi(X)=X_{0}+\cdots+X_{D}
$$

Теорема. Отображение $\psi$ - это точное представление алгебры Ли aut $_{0} M_{0}$ в алгебре Ли aut $_{0} Q_{0}$. 
ДокАзАтельство. Линейность очевидна; (d) означает, что если $X \in \mathrm{aut}_{0} M_{0}$, то $\psi(X) \in \operatorname{aut}_{0} Q_{0}$, точность следует из (е). Осталось проверить, что $\psi$ коммутирует с операцией взятия скобки полей. Действительно, пусть

$$
X=X_{0}+\cdots+X_{D}+\cdots \quad \text { и } \quad Y=Y_{0}+\cdots+Y_{D}+\cdots \in \text { aut }_{0} M_{0},
$$

тогда

$$
[X, Y]=\left[X_{0}+\cdots+X_{D}, Y_{0}+\cdots+Y_{D}\right]+\text { компоненты веса выше, чем } D,
$$

и, тем самым, $\psi([X, Y])=[\psi(X), \psi(Y)]$.

СлеДСтвиЕ. (а) Алгебра Ли автоморфизмов произвольного вполне невырожденного ростка aut $M_{\xi}$ изоморфна некоторой подалгебре Ли алгебры Ли aut $_{0} Q_{0}$ ее касательной моделъной поверхности $Q=Q\left(M_{\xi}\right)$.

(b) Группа Ли $\mathrm{Aut}_{\xi} M_{\xi}$ изоморфна некоторой подгруппе группы Ли $\mathrm{Aut}_{0} Q_{0}$.

Группа Aut $M_{\xi}$ представляет собой связную компоненту единицы в полном стабилизаторе точки. По-видимому верно, что не только связная компонента единицы, но и весь стабилизатор изоморфен некоторой подгруппе $\mathrm{Aut}_{0} Q_{0}$. Для гиперповерхности это утверждение является очевидным следствием теоремы Белошапки-Лободы [4]. Для многообразий длины три $(\ell=3)$ это следует из теоремы Коссовского [5], который доказал, что у таких многообразий в стабилизаторе нет нелинейных преобразований. Если гипотеза об отсутствии нелинейных преобразований в стабилизаторе верна и для многообразий с $\ell \geqslant 4$, то теорема о вложении справедлива для полного стабилизатора и для этих многообразий. Однако в общем случае доказательство этого утверждения для полного стабилизатора требует дополнительных рассуждений.

\section{СПИСОК ЦИТИРОВАННОЙ ЛИТЕРАТУРЫ}

[1] В.К. Белошапка, "Вещественные подмногообразия комплексного пространства их полиномиальные модели, автоморфизмы и проблемы классификации", УМH, 57:1 (2002), 3-44.

[2] В.К. Белошапка, "Универсальная модель вещественного подмногообразия", Матем. заметки, 75:4 (2004), 507-522.

[3] M.S. Baouendi, L. P. Rothschild, J. Winkelmann, D. Zaitsev, "Lie group structures of diffeomorphisms and applications to CR manifolds", Ann. Inst. Fourier (Grenoble), $\mathbf{5 4}$ (2004), 1279-1303.

[4] А. Г. Витушкин, "Голоморфные отображения и геометрия поверхностей", Современные проблемы математики. Фундаментальные направления, 7, ВИНИТИ, М., 1985, $167-226$.

[5] Р. В. Гаммель, И. Г. Коссовский, “Оболочка голоморфности модельной поверхности степени три и феномен "жесткости"”, Комплексныц анализ и приложения: Сборник статей, Тр. МИАН, 253, 2006, 30-45. 